\title{
MAGNETIC CURVES CORRESPONDING TO KILLING MAGNETIC FIELDS IN $\mathbb{E}^{3}$
}

\author{
SIMONA LUIZA DRUŢĂ-ROMANIUC AND MARIAN IOAN MUNTEANU
}

\begin{abstract}
We explicitly determine all magnetic curves corresponding to the Killing magnetic fields on the 3-dimensional Euclidean space.

Keywords and Phrases. Killing magnetic field, Lorentz force, magnetic curve. 2010 MSC: 53A04, 65D17
\end{abstract}

\section{INTRODUCTION}

The geodesic flow on a Riemannian manifold represents the extremals of the least action principle, namely it is determined by the motion of a certain physical system in the manifold. It is known that the geodesic equations are second order non-linear differential equations and they usually appear in the form of Euler-Lagrange equations of motion. Magnetic curves generalize geodesics. In physics, such a curve represents a trajectory of a charged particle moving on the manifold under the action of the magnetic field.

Let $(M, g)$ be an $n$-dimensional Riemannian manifold. A magnetic field is a closed 2-form $F$ on $M$ and the Lorentz force of a magnetic field $F$ on $(M, g)$ is an $(1,1)$ tensor field $\Phi$ given by

$$
g(\Phi(X), Y)=F(X, Y), \quad \forall X, Y \in \chi(M) .
$$

The magnetic trajectories of $F$ are curves $\gamma$ on $M$ that satisfy the Lorentz equation (sometimes called the Newton equation)

$$
\nabla_{\gamma^{\prime}} \gamma^{\prime}=\Phi\left(\gamma^{\prime}\right)
$$

Lorentz equation generalizes the equation satisfied by the geodesics of $M$, namely

$$
\nabla_{\gamma^{\prime}} \gamma^{\prime}=0
$$

Therefore, from the point of view of the dynamical systems, a geodesic corresponds to a trajectory of a particle without an action of a magnetic field, while a magnetic trajectory is a flowline of the dynamical system, associated with the magnetic field.

Since the Lorentz force is skew symmetric we have

$$
\frac{d}{d t} g\left(\gamma^{\prime}, \gamma^{\prime}\right)=2 g\left(\nabla_{\gamma^{\prime}} \gamma^{\prime}, \gamma^{\prime}\right)=0
$$

so the magnetic curves (trajectories) have constant speed $v(t)=\left\|\gamma^{\prime}\right\|=v_{0}$. When the magnetic curve $\gamma(t)$ is arc length parametrized $\left(v_{0}=1\right)$, it is called a normal magnetic curve.

Recall that a vector field $V$ on $M$ is Killing if and only if it satisfies the Killing equation:

$$
g\left(\nabla_{Y} V, Z\right)+g\left(\nabla_{Z} V, Y\right)=0
$$

for every vector fields $Y, Z$ on $M$, where $\nabla$ is the Levi Civita connection on $M$. 
A typical example of uniform magnetic fields is obtained by multiplying the volume form on a Riemannian surface by a scalar $s$ (usually called strength). When the surface is of constant Gaussian curvature $K$, trajectories of such magnetic fields are well known. More precisely, on the sphere $\mathbb{S}^{2}(K), K>0$, trajectories are small (Euclidean) circles of radius $\left(s^{2}+K\right)^{-1 / 2}$, on the Euclidean plane they are circles and the period of motion equals to $\frac{2 \pi}{s}$, while, on a hyperbolic plane $\mathbb{H}^{2}(-K), K>0$, trajectories can be either closed curves (when $|s|>\sqrt{K})$, or open curves. Moreover, when $|s|=\sqrt{K}$ normal trajectories are horocycles (see e.g. [11, 12]).

This problem was extended also for different ambient spaces. For example, if the ambient is a complex space form, Kähler magnetic fields are studied (see [2]), in particular, explicit trajectories for Kähler magnetic fields are found in the complex projective space $\mathbb{C P}^{n}$ [1. Kähler magnetic fields appear in theoretical and mathematical physics, varying from quantum field theory and string theory to general relativity.

If the ambient is a contact manifold, the fundamental 2-form defines the so-called contact magnetic field. Interesting results are obtained when the manifold is Sasakian, namely the angle between the velocity of a normal magnetic curve and the Reeb vector field is constant (see [9]). Moreover, explicit description for normal flowlines of the contact magnetic field on a 3-dimensional Sasakian manifold is known [9].

In the case of a 3 -dimensional Riemannian manifold $(M, g), 2$-forms and vector fields may be identified via the Hodge star operator $\star$ and the volume form $d v_{g}$ of the manifold. Thus, magnetic fields mean divergence free vector fields (see e.g. [10]). In particular, Killing vector fields define an important class of magnetic fields, called Killing magnetic fields. It is known that geodesics can be defined as extremal curves for the action energy functional. A variational approach to describe Killing magnetic flows in spaces of constant curvature is given in [7].

Note that, one can define on $M$ the cross product of two vector fields $X, Y \in \chi(M)$ as follows

$$
g(X \times Y, Z)=d v_{g}(X, Y, Z), \quad \forall Z \in \chi(M) .
$$

If $V$ is a Killing vector field on $M$, let $F_{V}=\iota_{V} d v_{g}$ be the corresponding Killing magnetic field. By $\iota$ we denote the inner product. Then, the Lorentz force of $F_{V}$ is (see [10])

$$
\Phi(X)=V \times X .
$$

Consequently, the Lorentz force equation (2) can be written as

$$
\nabla_{\gamma^{\prime}} \gamma^{\prime}=V \times \gamma^{\prime} .
$$

In what follows we consider the 3 -dimensional Euclidian space $\mathbb{E}^{3}$, endowed with the usual scalar product $\langle$,$\rangle .$

The fundamental solutions of (3) are $\left\{\partial_{x}, \partial_{y}, \partial_{z},-y \partial_{x}+x \partial_{y},-z \partial_{y}+y \partial_{z}, z \partial_{x}-x \partial_{z}\right\}$ and they give a basis of Killing vector fields on $\mathbb{E}^{3}$. Here $x, y, z$ denote the global coordinates on $\mathbb{E}^{3}$ and $\mathbb{R}^{3}=\operatorname{span}\left\{\partial_{x}, \partial_{y}, \partial_{z}\right\}$ is regarded as a vector space.

The easiest example is to consider the Killing vector field $\xi_{0}=\partial_{z}$. (Similar discussions can be made for $\partial_{x}$ and $\partial_{y}$, respectively.) Its trajectories are helices with axis $\partial_{z}$, namely $t \mapsto\left(x_{0}+a \cos t, y_{0}+a \sin t, z_{0}+b t\right)$, where $\left(x_{0}, y_{0}, z_{0}\right) \in \mathbb{R}^{3}$ and $a, b \in \mathbb{R}$. An interesting fact is that Lancret curves (i.e. general helices) in $\mathbb{E}^{3}$ are characterized by the following property (in our framework): they are magnetic trajectories associated with magnetic fields parallel to their axis. A similar result, relating Killing magnetic fields and Lancret curves is provided 
on the 3-sphere (see e.g. [7). Theorems of Lancret for general helices in 3-dimensional real space forms are presented in 3 .

In this paper we consider the following magnetic field $F_{V}=-(x d x+y d y) \wedge d z$ in $\mathbb{E}^{3}$, determined by the Killing vector field $V=-y \partial_{x}+x \partial_{y}$. The other two rotational vector fields $-z \partial_{y}+y \partial_{z}$ and $z \partial_{x}-x \partial_{z}$ give rise to analogue classifications for corresponding magnetic trajectories. The aim of this note is to find all magnetic curves corresponding to $F_{V}$. The main result we obtain is the following:

Theorem. The magnetic trajectories of the Killing magnetic field $F_{V}$ are: (a) planar curves situated in a vertical strip; (b) circular helices and (c) curve parametrized by

$$
x(t)=\rho(t) \cos \phi(t), y(t)=\rho(t) \sin \phi(t), z(t)=-\frac{1}{2} \int^{t} \rho^{2}(\zeta) d \zeta
$$

where $\rho$ and $\phi$ satisfy

$$
\left(\frac{d \rho^{2}}{d t}\right)^{2}+P\left(\rho^{2}(t)\right)=0, \quad \rho^{2}(t) \phi^{\prime}(t)=\text { constant }
$$

and $P$ is a polynomial of degree 3 .

We are able to obtain explicit solutions in case (c) and we represent some examples by using numerical approximations for some integrals.

Recall, for later use, some basic facts on normal elliptic integral of the first kind (see for example [8]):

$$
\int_{0}^{y} \frac{d t}{\sqrt{\left(1-t^{2}\right)\left(1-k^{2} t^{2}\right)}}=\int_{0}^{\varphi} \frac{d \vartheta}{\sqrt{1-k^{2} \sin ^{2} \vartheta}}=u=\operatorname{sn}^{-1}(y, k)=F(\varphi, k),
$$

where $y=\sin \varphi$ and $\varphi=$ am $u$. The angle $\varphi$ is called Jacobi amplitude and the function sn in known as Jacobi elliptic sine. The number $k$ is called modulus and for applications to engineering and physics it belongs to $(0,1)$.

\section{Rotational MAGNETiC tRAJECTORIES IN $\mathbb{E}^{3}$}

Let us consider the Killing vector field $V=-y \partial_{x}+x \partial_{y}$ on $\mathbb{E}_{3} \backslash O z$, which defines the magnetic field $F_{V}=-(x d x+y d y) \wedge d z$. The Lorentz force $\Phi_{V}$ acts on the vector space $\mathbb{R}^{3}$ as follows:

$$
\Phi_{V} \partial_{x}=-x \partial_{z}, \Phi_{V} \partial_{y}=-y \partial_{z}, \Phi_{V} \partial_{z}=x \partial_{x}+y \partial_{y}
$$

For the Euclidian space $\mathbb{E}^{3}$ the Lorentz force equation becomes

$$
\gamma^{\prime \prime}=V \times \gamma^{\prime}
$$

where the curve $\gamma: I=[0, l] \longrightarrow \mathbb{E}^{3}, \gamma(t)=(x(t), y(t), z(t))$ is parametrized by arc length, namely

$$
x^{\prime}(t)^{2}+y^{\prime}(t)^{2}+z^{\prime}(t)^{2}=1, \quad \forall t \in I
$$

and at the moment $t=0$ it passes through the point $\left(x_{0}, y_{0}, z_{0}\right)$, with the velocity $\left(u_{0}, v_{0}, w_{0}\right)$, such that

$$
u_{0}^{2}+v_{0}^{2}+w_{0}^{2}=1
$$


Proof of the Theorem. Our aim is to determine the magnetic curves of $F_{V}$. The equation (41) yields the following ordinary differential equations system

$$
\left\{\begin{array}{l}
x^{\prime \prime}=x z^{\prime} \\
y^{\prime \prime}=y z^{\prime} \\
z^{\prime \prime}=-\left(x x^{\prime}+y y^{\prime}\right) .
\end{array}\right.
$$

In order to solve it, note that from the first two equations we get a prime integral

$$
x^{\prime} y-y^{\prime} x=u_{0} y_{0}-x_{0} v_{0}
$$

while from the third equation we obtain

$$
z^{\prime}=-\frac{1}{2}\left(x^{2}+y^{2}\right)+\frac{1}{2}\left(x_{0}^{2}+y_{0}^{2}\right)+w_{0} .
$$

Notice that $z^{\prime}$ cannot vanish identically (on a subinterval of $I$ ). Indeed, if $z^{\prime}=0$ then $x^{\prime}=u_{0}, y^{\prime}=v_{0}$ and $z=z_{0}$ with $u_{0}^{2}+v_{0}^{2}=1$. Hence, $x(t)=x_{0}+u_{0} t, y(t)=y_{0}+v_{0} t$ and combining with (8) we get a contradiction. It follows that one cannot have horizontal magnetic curves corresponding to $V$.

In the sequel it is more convenient to consider cylindrical coordinates $\{\rho, \phi, z\}$ on $\mathbb{E}^{3} \backslash O z$. Thus, for our curve we have

$$
\left\{\begin{array}{l}
x=\rho(t) \cos \phi(t) \\
y=\rho(t) \sin \phi(t) \\
z=z(t)
\end{array}\right.
$$

where $\rho^{2}(t)=x^{2}(t)+y^{2}(t), \rho(t) \gtrless 0$.

Case I. First we study the general case, when $z^{\prime}$ is not constant (equivalently $\rho$ is not constant). The relations (77) and (8) lead to

$$
\begin{gathered}
\rho^{2}(t) \phi^{\prime}(t)=p_{0} \\
z^{\prime}(t)=q_{0}-\frac{1}{2} \rho^{2}(t)
\end{gathered}
$$

where we put $p_{0}=x_{0} v_{0}-u_{0} y_{0}$ and $q_{0}=\frac{1}{2}\left(x_{0}^{2}+y_{0}^{2}\right)+w_{0}$.

The arc length parametrization condition (5), together with (10), becomes

$$
\rho^{\prime 2}(t)+\rho^{2}(t) \phi^{\prime 2}(t)+q_{0}^{2}-q_{0} \rho^{2}(t)+\frac{1}{4} \rho^{4}(t)=1 .
$$

Multiplying (11) by $4 \rho^{2}(t)$, using (9) and denoting $\rho^{2}(t)$ by $f(t)>0$, for all $t \in I$, one gets

$$
f^{\prime 2}+f^{3}-4 q_{0} f^{2}+4\left(q_{0}^{2}-1\right) f+4 p_{0}^{2}=0 .
$$

We start to study the above differential equation for some particular values of the constants $p_{0}$ and $q_{0}$.

If $p_{0}=0$, i.e. $x_{0} v_{0}=y_{0} u_{0}$ it follows that the angle $\phi$ is constant, $\phi=\phi_{0}$, so the magnetic trajectory is a planar curve, with

$$
x(t)=\rho(t) \cos \phi_{0}, y(t)=\rho(t) \sin \phi_{0} .
$$

More precisely, the curve lies in the plane $\left(\sin \phi_{0}\right) x-\left(\cos \phi_{0}\right) y=0$. The initial conditions expressed in cylindrical coordinates, may be written as

$$
x_{0}=\rho_{0} \cos \phi_{0}, y_{0}=\rho_{0} \sin \phi_{0}
$$


and the condition $x_{0} v_{0}=y_{0} u_{0}$ becomes $u_{0}=\zeta_{0} \cos \phi_{0}, v_{0}=\zeta_{0} \sin \phi_{0}$, for a certain $\zeta_{0} \in \mathbb{R}$. It follows that $\zeta_{0}^{2}+\left(q_{0}-\frac{1}{2} \rho_{0}^{2}\right)^{2}=1$

$$
-1+\frac{1}{2} \rho_{0}^{2} \leq q_{0} \leq 1+\frac{1}{2} \rho_{0}^{2}
$$

Since $\rho_{0} \gtrless 0$ it follows that $q_{0}>-1$.

Let us solve the equation (12), for three particular situations arising from the initial conditions:

(i) If $p_{0}=0$ and $q_{0}=0$, then the equation (12) takes the form

$$
f^{\prime 2}(t)+f(t)(f(t)-2)(f(t)+2)=0
$$

and it has solution if and only if $f(t) \leq 2$, i.e. $\rho(t) \in(0, \sqrt{2}]$, so the magnetic curve $\gamma$ lies inside a cylinder. In fact, being a planar curve, $\gamma$ stays in a vertical strip centered in $O z$ and of width $2 \sqrt{2}$.

We have $f^{\prime}(t)= \pm \sqrt{f(t)\left(4-f(t)^{2}\right)}$ and we consider only the plus sign (the other situation may be treated in similar way). Supposing $\rho_{0} \neq \sqrt{2}$, we have that $f$ and the integral $\mathcal{I}(f)=\int_{\rho_{0}^{2}}^{f} \frac{d \zeta}{\sqrt{\zeta\left(4-\zeta^{2}\right)}}$ are strictly increasing functions. Thus, the equation $\mathcal{I}(f)=t$ has a unique solution in the interval $\left(\rho_{0}^{2}, 2\right)$, namely $f=\mathcal{J}(t)$, where $\mathcal{J}$ is the inverse function of $\mathcal{I}$. Consequently, $\rho(t)=\sqrt{\mathcal{J}(t)}$. In fact $\mathcal{J}$ may be expressed in terms of the elliptic functions. More precisely,

$$
\mathcal{J}(t)=\frac{2 \operatorname{sn}^{2}\left(t+t_{0}, \frac{1}{\sqrt{2}}\right)}{2-\operatorname{sn}^{2}\left(t+t_{0}, \frac{1}{\sqrt{2}}\right)}
$$

where $t_{0}$ is determined by $\operatorname{sn}\left(t_{0}, \frac{1}{\sqrt{2}}\right)=\frac{\sqrt{2} \rho_{0}}{\sqrt{2+\rho_{0}^{2}}}$.

Summarizing, the magnetic curve is given by

$$
x(t)=\sqrt{\mathcal{J}(t)} \cos \phi_{0}, y(t)=\sqrt{\mathcal{J}(t)} \sin \phi_{0}, z(t)=-\frac{1}{2} \int_{0}^{t} \mathcal{J}(\zeta) d \zeta .
$$

In order to draw a picture of our curve, one can use Matlab to compute the parametrization. The idea is to calculate the integrals numerically, as Riemann sums. See Appendix.

(ii) If $p_{0}=0, q_{0}=1$, then the equation (12) becomes $f^{\prime 2}(t)+f^{2}(t)(f(t)-4)=0$, from which we have that $f(t) \leq 4$, equivalently $\rho(t) \leq 2$, so the magnetic curve $\gamma$ stays inside a cylinder of radius 2 . In fact, being planar, the curve lies in a vertical strip centered on $z$-axis. The equation can be written in the form

$$
\frac{d f}{f \sqrt{4-f}}= \pm d t \text {. }
$$

Taking the plus sign, one gets the solution

$$
f(t)=\frac{4}{\cosh ^{2}\left(t-t_{0}\right)}, \quad t \in\left(0, t_{0}\right)
$$

where $t_{0}=-\frac{1}{2} \ln \frac{2-\sqrt{4-\rho_{0}^{2}}}{2+\sqrt{4-\rho_{0}^{2}}}$. Hence

$$
\rho(t)=\frac{2}{\cosh \left(t-t_{0}\right)}
$$


and the magnetic curve is parametrized by

$$
x(t)=\frac{2 \cos \phi_{0}}{\cosh \left(t-t_{0}\right)}, y(t)=\frac{2 \sin \phi_{0}}{\cosh \left(t-t_{0}\right)}, z(t)=z_{0}+t-2\left(\tanh \left(t-t_{0}\right)+\tanh t_{0}\right) .
$$

We draw a picture of this (planar) curve.

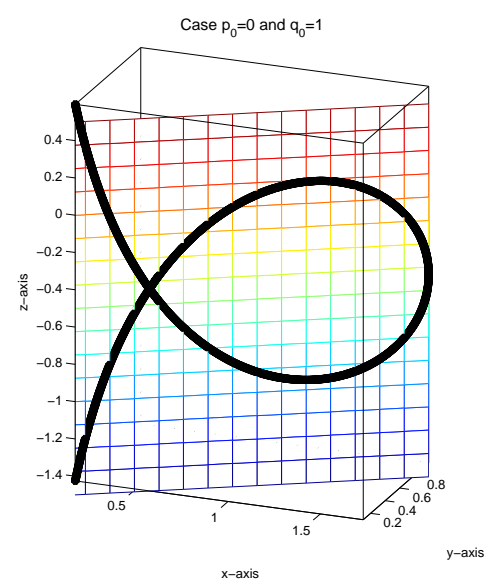

FigurE 1. $t \in \mathbb{R}, \phi_{0}=\frac{\pi}{6}$

Let us finalize the examination of the equation (12) for $p_{0}=0$. The polynomial $P(f)=f\left(f^{2}-4 q_{0} f+4\left(q_{0}^{2}-1\right)\right)$ has three real solutions, namely $f_{1}=2\left(q_{0}-1\right), f_{2}=2\left(q_{0}+1\right)$ and $f_{3}=0$. If $f$ is a solution for (12), then $P(f)$ should be negative. Recall that $q_{0}>-1$. We have

(a) If $q_{0} \in(-1,1)$, then $f_{1}<0<f_{2}$. It follows that $\rho(t) \in\left(0, \sqrt{2\left(q_{0}+1\right)}\right)$ and the discussion is similar as in case $q_{0}=0$. More precisely we have

$$
\rho(t)^{2}=\frac{2\left(1-q_{0}^{2}\right) \operatorname{sn}^{2}\left(t+t_{0}, \sqrt{\frac{q_{0}+1}{2}}\right)}{2-\left(q_{0}+1\right) \operatorname{sn}^{2}\left(t+t_{0}, \sqrt{\frac{q_{0}+1}{2}}\right)}
$$

where $t_{0}$ is defined by $\operatorname{sn}\left(t_{0}, \sqrt{\frac{q_{0}+1}{2}}\right)=\sqrt{\frac{2}{q_{0}+1}} \frac{\rho_{0}}{\sqrt{\rho_{0}^{2}-2\left(q_{0}-1\right)}}$.

(b) If $q_{0}>1$, then $0<f_{1}<f_{2}$. It follows that $f(t) \in\left(f_{1}, f_{2}\right)$. Thus, the curve $\gamma$ lies between two cylinders since $\rho(t) \in\left(\sqrt{2\left(q_{0}-1\right)}, \sqrt{2\left(q_{0}+1\right)}\right)$. As before, the curve is situated in a union of two vertical strips. Again, the discussion is similar as in case $q_{0}=0$. In terms of elliptic functions, we may write

$$
\rho(t)^{2}=\frac{q_{0}^{2}-1}{\frac{q_{0}+1}{2}-\operatorname{sn}^{2}\left(\sqrt{\frac{q_{0}+1}{2}} t+t_{0}, \sqrt{\frac{2}{q_{0}+1}}\right)}
$$

where $t_{0}$ is defined by $\operatorname{sn}\left(t_{0}, \sqrt{\frac{2}{q_{0}+1}}\right)=\sqrt{\frac{q_{0}+1}{2}} \frac{\sqrt{\rho_{0}^{2}-2\left(q_{0}-1\right)}}{\rho_{0}}$.

In order to visualize an example, consider the following initial conditions: $x_{0}=2$, $y_{0}=0, z_{0}=0$ and $u_{0}=0, v_{0}=0, w_{0}=1$ (this yields $p_{0}=0$ and $q_{0}=3$ ). 
We will use again Matlab to compute the integrals (numerically) and to draw the picture.

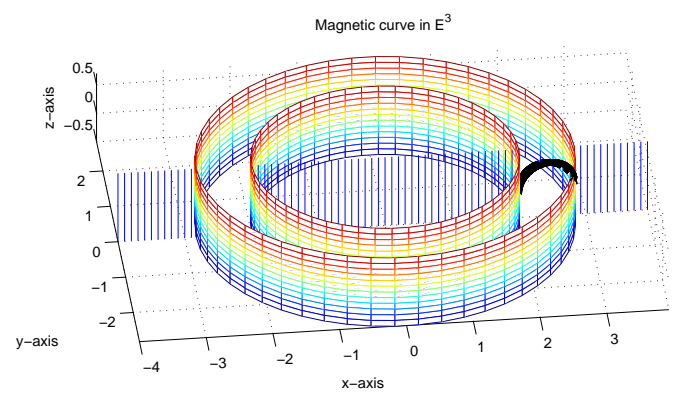

FiguRE 2. $p_{0}=0, q_{0}=3$

Return to (12) for $p_{0} \neq 0$ and notice that the equation

$$
P(f)=f^{3}-4 q_{0} f^{2}+4\left(q_{0}^{2}-1\right) f+4 p_{0}^{2}=0
$$

has the discriminant

$$
\Delta=-16\left[27 p_{0}^{4}+8 p_{0}^{2} q_{0}\left(q_{0}^{2}-9\right)-16\left(q_{0}^{2}-1\right)^{2}\right]
$$

and the following situations appear:

- the equation (13) has three distinct solutions iff $\Delta>0$.

- the polynomial $P$ has multiple roots iff $\Delta=0$.

- the polynomial $P$ has one real root and two complex conjugate roots iff $\Delta<0$.

A detailed analysis of the above situations, lead us to conclude, after taking into account classical Viète's formulas, that the equation (12) has solutions if and only if $\Delta>0$.

Indeed, if $\Delta<0$, let $A \in \mathbb{C} \backslash \mathbb{R}$ and $\bar{A}$ be the complex solutions of (13), and $B$ its real solution. Then, the ODE (12) can be rewritten as

$$
f^{\prime}(t)^{2}+\left(f(t)^{2}-2 \operatorname{Re}(A) t+|A|^{2}\right)(f(t)-B)=0,
$$

where $\operatorname{Re}(A)$ denotes the real part of the complex number $A$. From the third Viète's formula we conclude that $B$ should be negative, and consequently, the previous equality cannot occur.

On the other hand, if $\Delta=0$, analyzing the coefficients one cannot have a triple root (since $16 q_{0}^{2} \neq 12\left(q_{0}^{2}-1\right)$. Hence, let $A \in \mathbb{R}$ be the double root, and let $B \in \mathbb{R}$ be the third one. With a similar argument as above, $B$ is negative and the ODE (12) becomes

$$
f^{\prime}(t)^{2}+(f(t)-A)^{2}(f(t)-B)=0 .
$$

Again, this equality cannot hold.

It follows that $\Delta$ should be (strictly) positive. Let $A, B, C \in \mathbb{R}$ be the three distinct solutions of (13). The third Viète's formula yields $A B C=-4 p_{0}^{2}<0$, and hence 
a) either $A, B, C$ are all negative,

b) or two of them, $A$ and $B$, are positive and the third one, $C$, is negative.

In case a) the ODE (12): $f^{\prime}(t)^{2}+(f(t)-A)(f(t)-B)(f(t)-C)=0$ has no solution. This happens if and only if $q_{0}<-1$ and $p_{0} \neq 0$ (for the proof use the second and the third Viète's formulas) together with $\Delta>0$ (for example if $q_{0}=-3$ and $p_{0}=1$ ).

In case b), equivalently to $\Delta>0, q_{0}>-1$ and $p_{0} \neq 0$, the equation (12) has a solution in the interval defined by the positive solutions $A<B$ of (13). Since the function $\mathcal{I}(f)=\int_{A}^{f} \frac{d \zeta}{\sqrt{(\zeta-A)(B-\zeta)(\zeta-C)}}$ is strictly increasing, $\mathcal{I}(f)=t$ has a unique solution $f$, denoted by $\mathcal{J}(t)$. Thus we have $\rho=\sqrt{\mathcal{J}(t)}$, and $\phi(t)=\phi_{0}+p_{0} \int_{0}^{t} \frac{d \zeta}{\mathcal{J}(\zeta)}$. In this case, the magnetic curve $\gamma$ is given by

$$
\begin{gathered}
x(t)=\sqrt{\mathcal{J}(t)} \cos \left(\phi_{0}+p_{0} \int_{0}^{t} \frac{d \zeta}{\mathcal{J}(\zeta)}\right), y(t)=\sqrt{\mathcal{J}(t)} \sin \left(\phi_{0}+p_{0} \int_{0}^{t} \frac{d \zeta}{\mathcal{J}(\zeta)}\right), \\
z(t)=z_{0}+q_{0} t-\frac{1}{2} \int_{0}^{t} \mathcal{J}(\zeta) d \zeta .
\end{gathered}
$$

We may express $\rho$ in terms of elliptic functions, namely

$$
\rho(t)^{2}=\frac{A k^{2}-C \mathrm{sn}^{2}\left(r t+t_{0}, \frac{1}{k}\right)}{k^{2}-\operatorname{sn}^{2}\left(r t+t_{0}, \frac{1}{k}\right)}
$$

where $k^{2}=\frac{B-C}{B-A}, r=\frac{\sqrt{B-C}}{2}$, and $\operatorname{sn}\left(t_{0}, \frac{1}{k}\right)=k \sqrt{\frac{\rho_{0}^{2}-A}{\rho_{0}^{2}-C}}$.

In the Appendix we will draw a picture (using the same technique in Matlab as before) corresponding to the following data: $p_{0}=\frac{\sqrt{2 \sqrt{6}-3}}{2}, q_{0}=\frac{3-\sqrt{6}}{2}$, for which we have $A=1$, $B=2$ and $C=3-2 \sqrt{6}$.

The situation $I(f)=-t$ can be treated in similar way.

Finally, notice that for $q_{0}=-1$ we get $\Delta=-16 p_{0}^{2}\left(27 p_{0}^{2}+64\right)$ and this case was discussed above.

Case II. Now, let us study the remaining case when $z^{\prime}(t)=w_{0} \neq 0$. We immediately have that

and from (8) we obtain

$$
z(t)=z_{0}+t w_{0},
$$

$$
x^{2}+y^{2}=x_{0}^{2}+y_{0}^{2} .
$$

This means that the magnetic trajectory $\gamma$ lies on the circular cylinder of radius $\rho_{0}=\sqrt{x_{0}^{2}+y_{0}^{2}}$.

Two subcases must be discussed: $w_{0}<0$ and $w_{0}>0$.

II.1: In the case when $w_{0}<0$ the magnetic curve is given by

$$
\left\{\begin{array}{l}
x(t)=x_{0} \cos \left(\sqrt{-w_{0}} t\right)+\frac{u_{0}}{\sqrt{-w_{0}}} \sin \left(\sqrt{-w_{0}} t\right) \\
y(t)=y_{0} \cos \left(\sqrt{-w_{0}} t\right)+\frac{v_{0}}{\sqrt{-w_{0}}} \sin \left(\sqrt{-w_{0}} t\right) \\
z(t)=z_{0}+t w_{0} .
\end{array}\right.
$$

This curve is a helix around the above cylinder. 
At this point, we have to find which are the initial conditions leading this situation. To do this, using (14) and (15), we should have the following relations

$$
w_{0}=-\frac{2}{\rho_{0}^{2}+\sqrt{\rho_{0}^{4}+4}}, u_{0}=\varepsilon \rho_{0} \sqrt{-w_{0}} \sin \phi_{0}, v_{0}=-\varepsilon \rho_{0} \sqrt{-w_{0}} \cos \phi_{0}
$$

where $\rho_{0}$ and $\phi_{0}$ have the usual meaning and $\varepsilon= \pm 1$.

II.2: If $w_{0}>0$, the ODE system (6) has the following solution

$$
\left\{\begin{array}{l}
x(t)=x_{0} \cosh \left(\sqrt{w_{0}} t\right)+\frac{u_{0}}{\sqrt{w_{0}}} \sinh \left(\sqrt{w_{0}} t\right) \\
y(t)=y_{0} \cosh \left(\sqrt{w_{0}} t\right)+\frac{v_{0}}{\sqrt{w_{0}}} \sinh \left(\sqrt{w_{0}} t\right) \\
z(t)=z_{0}+t w_{0}
\end{array}\right.
$$

but in this case the condition (14) is satisfied if and only if $x_{0}=y_{0}=0$ and $u_{0}=v_{0}=0$. This situation cannot occur.

\section{REVIEW ON THE CLASSiCAL MAGNETIC FIELD ON $\mathbb{E}^{3}$}

As we have already said in Introduction, the best known example of magnetic fields in the Euclidean space $\mathbb{E}^{3}$ is furnished by the 2 -form $F_{0}=d x \wedge d y$, corresponding to the Killing vector field $\xi_{0}=\frac{\partial}{\partial z}$.

In this section we consider the Killing magnetic field $F_{\xi}=s F_{0}=s d x \wedge d y$, determined by the Killing vector field $\xi=s \xi_{0}=s \partial_{z}$ on $\mathbb{E}^{3}$, where $s \neq 0$ is an arbitrary constant. We briefly describe its magnetic curves.

The action of the Lorentz force $\Phi_{\xi}$ on the vector space $\mathbb{R}^{3}$ is given by:

$$
\Phi_{\xi} \partial_{x}=s \partial_{y}, \Phi_{\xi} \partial_{y}=-s \partial_{x}, \Phi_{\xi} \partial_{z}=0 .
$$

Solving the Lorentz force equation $\gamma_{s}^{\prime \prime}=\Phi_{\xi}\left(\gamma_{s}^{\prime}\right)$, we obtain the family of magnetic curves $\gamma_{s}(t)=(x(t), y(t), z(t))$, parametrized by

$$
\left\{\begin{array}{l}
x(t)=\frac{u_{0}}{s} \sin (s t)+\frac{v_{0}}{s} \cos (s t)+x_{0}-\frac{v_{0}}{s} \\
y(t)=-\frac{u_{0}}{s} \cos (s t)+\frac{v_{0}}{s} \sin (s t)+y_{0}+\frac{u_{0}}{s} \\
z(t)=w_{0} t+z_{0} .
\end{array}\right.
$$

Write the first Frénet equation

$$
\gamma^{\prime \prime}=\kappa N
$$

where $\kappa$ is the curvature and $N$ is the normal of the curve. Using the equation (4) we obtain that the square of the curvature is

$$
\kappa^{2}=s^{2}\left(1-w_{0}^{2}\right) .
$$

Moreover, classical computations give the torsion $\tau=s w_{0}$.

Notice that even both the curvature $\kappa$ and the torsion $\tau$ depend on the strength $s$, the ratio $\frac{\tau}{\kappa}$ does not.

We conclude with some comments:

i) If $w_{0}=0$ the curvature is $\kappa=s$ and the torsion is $\tau=0$, so the magnetic line is a (planar) circle.

ii) If $w_{0}= \pm 1$, then $\kappa=0, \tau= \pm s$, so the magnetic curves are vertical lines.

iii) In other cases the magnetic curves are circular helices. 


\section{Appendix}

In this section we present a Matlab program in order to compute, by numerical approximation of the involved integrals, the parametrization of magnetic curve obtained in case I (i) from page 5. Since the curve is planar we consider $\phi_{0}=0$.

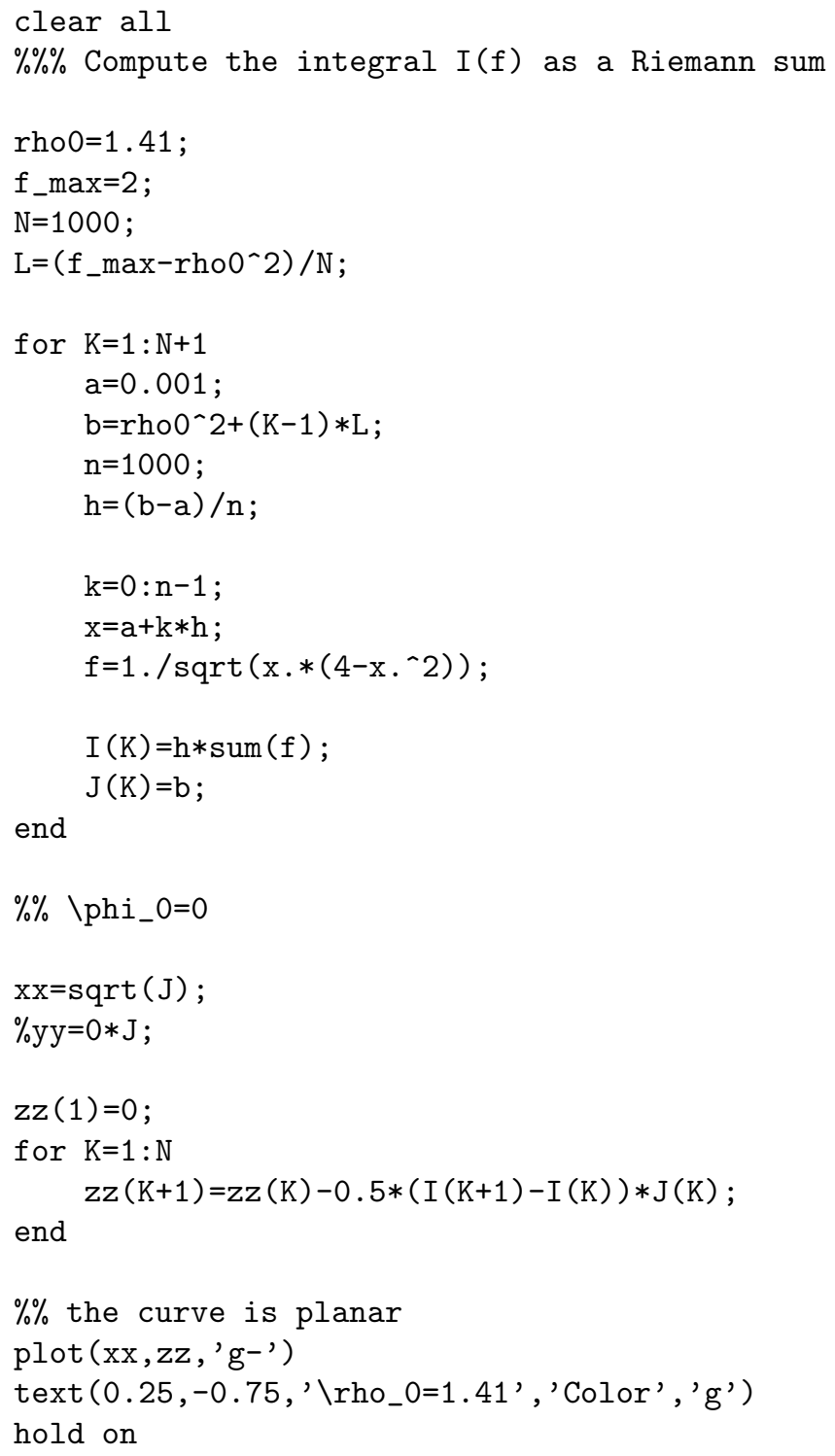

Representation of the magnetic curves depending on the initial position: 


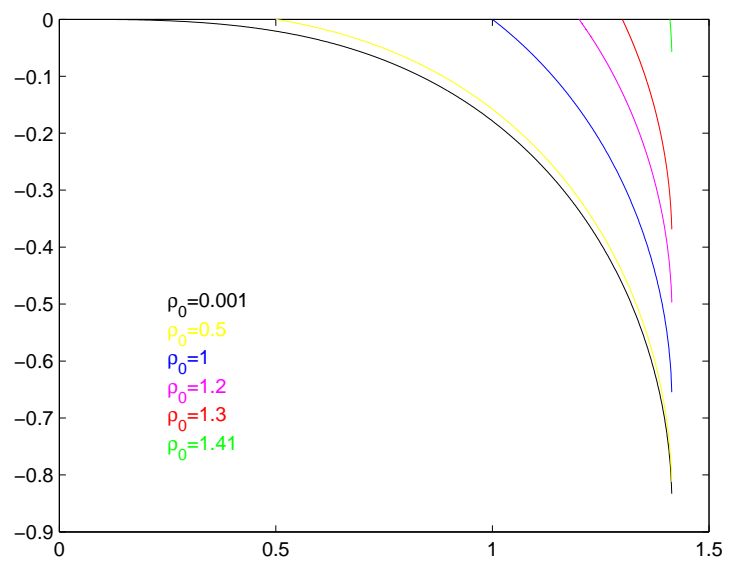

Figure 3. I (i)

Using the previous Matlab program adapted to the example furnished at page 8 , and for the initial data $\phi_{0}=0$ and $z_{0}=0$, we can represent the corresponding magnetic curves:

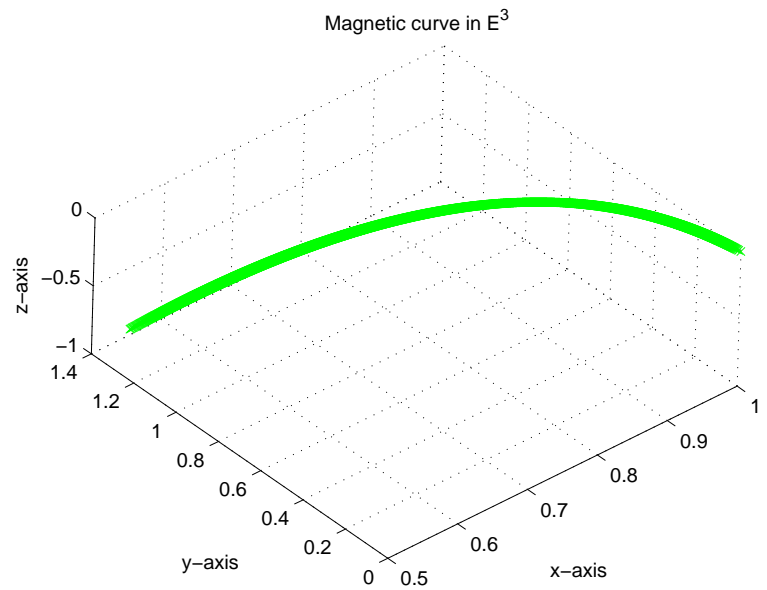

FIGURE 4. $x_{0}=1, y_{0}=0, z_{0}=0, u_{0}=0, v_{0}=\sqrt{\frac{2 \sqrt{6}-3}{2}}$ and $w_{0}=\frac{2-\sqrt{6}}{2}$

Acknowledgements. The first authors is a postdoctoral researcher in the framework of the program POSDRU 89/1.5/S/49944, 'AL. I. Cuza' University of Iaşi, Romania. The second author is supported by a Fulbright Grant no. 498 at the Michigan State University, USA.

\section{REFERENCES}

[1] Adachi, T., Kähler Magnetic Field on a Complex Projective Space, Proc. Japan Acad. 70 Ser. A (1994), $12-13$.

[2] Adachi, T., Kähler Magnetic Flow for a Manifold of Constant Holomorphic Sectional Curvature, Tokyo J. Math. 18 (1995) 2, 473-483. 
[3] Barros, M., General Helices and a Theorem of Lancret, Proc. AMS 125 (1997) 5, 1503-1509.

[4] Barros, M., Cabrerizo, J. L., Fernández, M., and Romero, A., Magnetic vortex filament flows, J. Math. Phys. 48 (2007) 8, 082904:1-27.

[5] Barros, M. and Ferrández, A., A conformal variational approach for helices in nature, J. Math. Phys. 50 (2009) 10, 103529:1-20.

[6] Barros, M., Romero, A., Cabrerizo, J. L., and Fernández, M., The Gauss-Landau-Hall problem on Riemannian surfaces, J. Math. Phys. 46 (2005), 112905:1-15.

[7] Barros, M. and Romero, A., Magnetic vortices, EPL 77 (2007), 34002:1-5.

[8] Byrd, P. F., Friedman, M. D., Handbook of Elliptic Integrals foe Engineers and Scientists, 2nd Edition revised, Springer, 1971.

[9] Cabrerizo, J. L., Fernández, M., and Gómez, J. S., On the existence of almost contact structure and the contact magnetic field, Acta Math. Hungar., 125 (2009)(1-2), 191-199.

[10] Cabrerizo, J. L., Fernández, M., and Gómez, J. S., The contact magnetic flow in $3 D$ Sasakian manifolds, J. Phys. A: Math. Theor., 42 (2009), 19, 195201:1-10.

[11] Comtet, A., On the Landau levels on the hyperbolic plane, Ann. of Phys. 173 (1987), 185-209.

[12] Sunada, T., Magnetic flows on a Riemann surface, Proceedings of KAIST Mathematics Workshop, 1993, 93-108.

(S.L. Druţă-Romaniuc) 'AL. I. Cuza' University of Iaşi, Department of Sciences, Lascăr Catargi Street, no. 54, 700107 Iaşi, Romania, email: simona.druta (at) uaic.ro

(M.I. Munteanu) 'Al.I.Cuza' University of Iaşi, Faculty of Mathematics, Bd. Carol I, no. 11, 700506 Iaşi, Romania, http://www.math.uaic.ro/ munteanu

(temporary address) Michigan State University, Department of Mathematics, Wells Hall, 48824-1029 East Lansing, USA, email: marian.ioan.munteanu (at) gmail.com 